\title{
New ultrashort pulsewidth measurement technology based on interference jitter and FPGA platform
}

\author{
Jin Li (李 瑾) $)^{1, \dagger}$, Yanbo Dou (窦妍博 $)^{1, \dagger}$ ， Lixin Wang (王立新) ${ }^{2}$, Jinhai Zou (邹金海) ${ }^{1}$, Yu Ding (丁 宇 $)^{2}$, Hang Wang (王 航) ${ }^{1}$, \\ Qiujun Ruan (阮秋君) ${ }^{1}$, Zhipeng Dong (董志鹏) ${ }^{1}$, and Zhengqian Luo (罗正钱) \\ ${ }^{1}$ Department of Electronic Engineering, Xiamen University, Xiamen 361005, China \\ ${ }^{2}$ Science and Technology on Electro-Optical Information Security Control Laboratory, Tianjin 300308, China
}

*Corresponding author: zqluo@xmu.edu.cn

Received November 15, 2021 | Accepted December 13, 2021 | Posted Online January 11, 2022

\begin{abstract}
Conventional ultrashort pulsewidth measurement technology is autocorrelation based on second-harmonic generation; however, nonlinear crystals and bulky components are required, which usually leads to the limited wavelength range and the difficult adjustment with free-space light alignment. Here, we proposed a compact all-fiber pulsewidth measurement technology based on the interference jitter ( $\mathrm{IJ}$ ) and field-programmable gate array (FPGA) platform, without requiring a nonlinear optical device [e.g., nonlinear crystal/detector). Such a technology shows a wide measurement waveband from 1 to $2.15 \mu \mathrm{m}$ at least, a pulsewidth range from femtoseconds to $100 \mathrm{ps}$, and a small relative error of $0.15 \%-3.8 \%$. In particular, a minimum pulse energy of $219 \mathrm{fJ}$ is experimentally detected with an average-power-peak-power product of $1.065 \times 10^{-6} \mathrm{~W}^{2}$. The IJ-FPGA technology may offer a new route for miniaturized, user-friendly, and broadband pulsewidth measurement.
\end{abstract}

Keywords: pulse width measurement; interference jitter; FPGA platform; optical device.

DOI: 10.3788/COL202220.031404

\section{Introduction}

Ultrafast lasers are widely used in many fields, such as biomedicine $^{[1]}$, material processing ${ }^{[2]}$, optical communications ${ }^{[3]}$, $\operatorname{Lidar}^{[4]}$, and scientific research ${ }^{[5,6]}$. Most of these applications need to clearly know the parameters (e.g., pulsewidth and phase) of ultrashort laser pulses, and it is important to accurately measure the pulse width and phase information of the ultrashort laser $^{[7]}$.

In 1967, the intensity autocorrelation (AC) theory was proposed $^{[8]}$, and $\mathrm{AC}$ based on second-harmonic generation (SHG) becomes one of the most key technologies to characterize ultrashort pulses. In 1993, the frequency-resolved optical gating (FROG) was proposed ${ }^{[9-13]}$, and then, in 1998, spectral-phase interferometry for direct electric-field reconstruction (SPIDER) was further developed ${ }^{[14-16]}$. These technologies, enabling retrieval of pulse amplitude and phase information simultaneously, have been a great success during the past few decades. However, they still suffer from some limitions $s^{[17,18]}$ : (1) the used nonlinear crystal restricts the measurement spectral range, leading to often changing nonlinear crystals for different wavebands; (2) precise phase matching must be satisfied by beam alignment in the free-space light path or temperature control, resulting in difficulty adjusting; (3) the measurement sensitivity is insufficient because enough power level is needed to excite the nonlinear process (e.g., SHG) ${ }^{[19]}$. In addition, another AC technology based on two-photon absorption (TPA) or saturable absorption allows compact design and high detection sensitivity $^{[20-24]}$, but the TPA effect usually has picosecond (ps) decay time in semiconductors, possibly leading to limitation on the measurement of pulse width ${ }^{[25,26]}$. In recent years, many researchers have proposed new pulsewidth measurement methods without the nonlinear crystal and TPA detector. For example, the linear interference to measure pulse width was already mentioned and confirmed in $2014^{[27]}$. The FROG method based on the transient grating nonlinear process was proposed in 2015, which does not need nonlinear crystals and self-phase matching $^{[28]}$. Self-referenced spectral interferometry and dispersion scan can be also run at low pulse energy without nonlinear crystals ${ }^{[29,30]}$. However, these methods are still relatively complex with a limited operation bandwidth. Therefore, there is still strong motivation to develop compact and broadband pulsewidth measurement technology without the use of nonlinear crystals/detectors.

In this Letter, we propose and demonstrate an all-fiber pulsewidth measurement technology based on the interference jitter (IJ) and field-programmable gate array (FPGA) platform, which 
does not require nonlinear crystals or a TPA detector and shows a compact/intelligent capability. The measured spectral range can cover the range from the near-infrared to mid-infrared, and the measured pulsewidth can be from tens of femtoseconds (fs) to $100 \mathrm{ps}$. This technology represents a new paradigm of pulsewidth measurement setup with the advantages of compactness, high performance, and ease of use.

\section{Operation Principle and Experimental Setup}

\subsection{Design and setup}

Figure 1 shows the schematic design of our proposed pulsewidth measurement technology based on IJ-FPGA. The measurement setup is mainly composed of an interference part and an IJ data acquisition and processing system. The interference part consists of two 50/50 optical couplers (OCs), a fixed arm with a section of matching fiber, and a fiber delay line (MDL-002, General Photonics, Inc.). The delay line is set to 0 ps position at absolute origin, and the delay range can be from 0 to $560 \mathrm{ps}$, which determines the maximum pulse width measured up to $\sim 280 \mathrm{ps}$. The optical delay resolution is $1 \mathrm{fs}$, and the optical delay accuracy is $\pm 10 \mathrm{fs}$, implying that the narrowest measurable pulsewidth is tens of fs. The IJ data processing system mainly realizes fast and accurate acquisition and recording of IJ signals at different delay $\tau$ (see Section 2.2). The system mainly includes the lowspeed photodetector (PD), carrier card, and factor card. The InGaAs amplified PD (Thorlabs, PDA05CF2) with a $150 \mathrm{MHz}$ bandwidth is used to measure the wavelength of $800-1700 \mathrm{~nm}$, and the noise equivalent power (NEP) is $12.6 \mathrm{pW} / \mathrm{Hz}^{1 / 2}$. In the $2 \mu \mathrm{m}$ band, there is a $12.5 \mathrm{GHz} \mathrm{PD}$ (Electro-Optics Technology, Inc., ET-5000F). The carrier card chooses the FPGA development board, and the factor card includes an analog-to-digital conversion (ADC) module and universal asynchronous receiver/transmitter (UART) transmission module. The ADC module realizes the conversion of the analog signal output stemming from the PD to the digital signal. The module has a sampling frequency of $125 \mathrm{MS} / \mathrm{s}$, a digital bit width of 12 bits, and an input voltage range of $-5-5 \mathrm{~V}$. The sampling frequency limits the repetition frequency of the signal source to be below $62.5 \mathrm{MHz}$, based on the Nyquist sampling

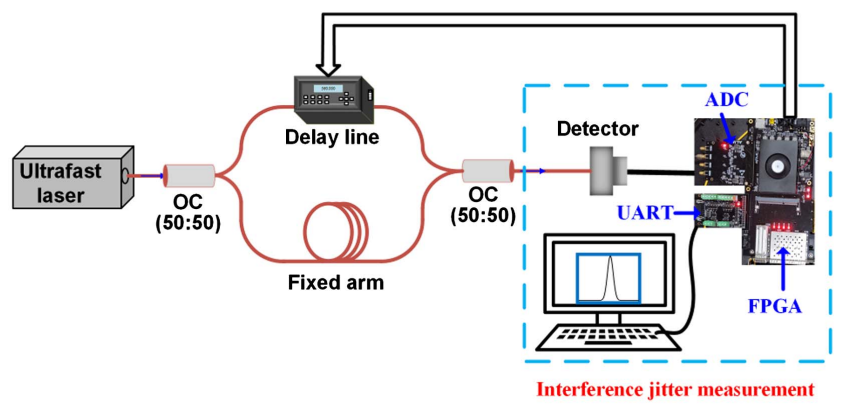

Fig. 1. Schematic of the all-fiber pulsewidth measurement setup based on IJ-FPGA. theorem. In our experiment, the IJ signal generally appears within $10 \mathrm{~s}$ after the delay-line set, so the FPGA carrier card mainly realizes the storage and maximum value detection of the digital signals collected within $10 \mathrm{~s}$. The UART module realizes the transmission of maximum value data to the personal computer (PC) with the transmission baud rate of 9600 . Since the method is a statistical detection method, it should be pointed out that our measurement method may only apply to the case of higher repetition rate [i.e., above the megahertz $(\mathrm{MHz})$ level].

\subsection{Operation principle}

According to the IJ-FPGA setup in Fig. 1, the ultrafast laser to be measured is divided into two arms, in which the delay time of one arm is variable, and the delay time of the other arm is fixed. The optical fields of the variable arm $E_{2}$ and the fixed arm $E_{1}$ interfere at the output port. After superposition, the optical field $E$ can be expressed as

$$
\begin{aligned}
E(\omega, t) & =E_{1}(\omega, t)+E_{2}(\omega, t-\tau) \\
& =A(t) e^{i[\omega t+\varphi(t)]}+A(t-\tau) e^{i[\omega(t-\tau)+\varphi(t-\tau)]},
\end{aligned}
$$

where $\tau$ is the delay time between the two arms, and $\omega$ and $\varphi(t)$ are the frequency and phase of the pulse, respectively. The photocurrent generated in the low-speed PD is too slow to capture either the envelope or the fast-oscillating fringes of an ultrashort pulse. The measured photocurrent must be the time integral of $|E(t)|^{2}$. When the delay time $\tau$ of the delay line is fixed, the phase difference from the two-arm pulses is still not fixed because of the external slowly varying environment (e.g., low-frequency vibration, temperature fluctuation), which finally reflects the slow jitter captured by the low-speed PD. Such a phenomenon can often be observed in an ultrafast time-domain interferometer, i.e., so-called IJ induced by the environmental disturbance. Figure 2(a) represents the typical states with different delay times $\tau$, which are non-overlapping, partial overlapping, and complete overlapping of the two-arm pulses, respectively. When the two-arm pulses are non-overlapping, no interference happens, and no jitter can be observed by the low-speed PD. With increasing overlapping of the two-arm pulses by scanning the delay line (varying $\tau$ ), the IJ becomes stronger and stronger, as described in Fig. 2(b). Next, we collect and record the IJ detected by the low-speed PD and find out the maximum output voltage $\left(V_{\max }\right)$ by the FPGA platform. Namely, we record $V_{\max }$ at each delay point $\tau$ and then draw the curve $\left(\tau, V_{\max }\right)$ given by the blue points of Fig. 2(c). By fitting, the full width at half-maximum (FWHM) of this curve is the measured pulse width $\Delta \tau^{\mathrm{FWHM}}$ (i.e., $\Delta \tau^{\mathrm{FWHM}}=\mathrm{FWHM}$ ). According to this idea and Eq. (1), we perform the numerical simulation. As shown in Fig. 3, the red curve is the optical intensity envelope of the original pulse, and the blue curve is the simulated envelope of $\left(\tau, V_{\max }\right)$. The two curves are consistent in both the ps and fs regimes, indicating the feasibility of the proposed technology. 

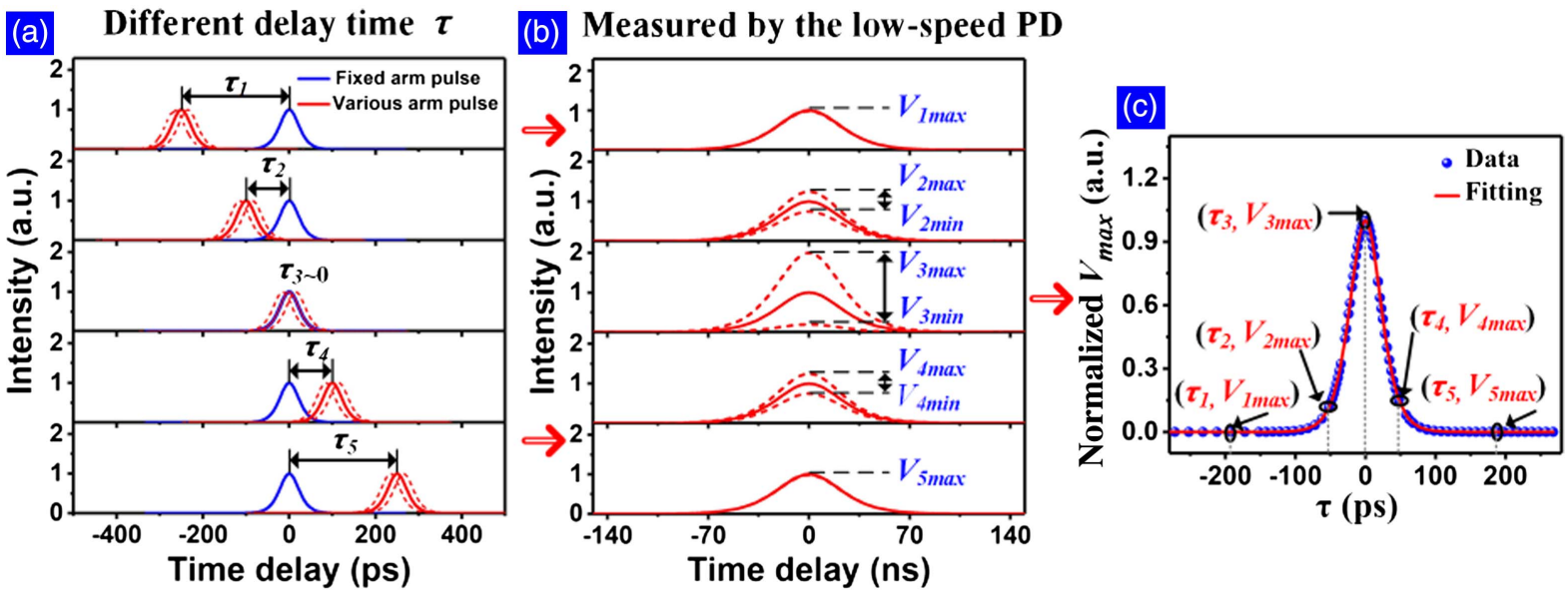

Fig. 2. (a) Pulses of the fixed arm and the variable arm at different delay times $\tau$, (b) the pulse envelope measured by a low-speed PD at the corresponding delay time $\tau_{\text {, and }}\left(\mathrm{c}\right.$ ) the normalized $V_{\max }$ data (blue dots) and fitting envelope (red line).

\section{Result and Discussion}

\subsection{Broadband pulsewidth measurement}

First, we used the IJ-FPGA setup (see Fig. 1) to measure $1.06 \mu \mathrm{m}$ ultrashort pulses, which are from a home-made, SESAM modelocked $\mathrm{Yb}$-doped polarization-maintaining fiber laser, as shown in Fig. 4(a). The spectrum [Fig. 4(b)] of the mode-locked laser has a central wavelength of $1064.0 \mathrm{~nm}$ and a pulse repetition rate of $19.331 \mathrm{MHz}$. For comparison, the pulse width was measured by our IJ-FPGA technology and a commercial autocorrelator, respectively. In Fig. 4(c), the red dots were recorded by the IJ-FPGA setup with the input average power of $15 \mu \mathrm{W}$, and $\Delta \tau^{\mathrm{FWHM}}$ was measured to be $11.74 \mathrm{ps}$. Meanwhile, the blue curve was recorded by a commercial autocorrelator (Femtochrome Research, FR-103XL), and the FWHM = 18.403 ps. Considering the hyperbolic secant pulse, the pulse width is $\Delta \tau^{\mathrm{FWHM}}=$ $\mathrm{FWHM} \times 0.648=11.95 \mathrm{ps}$. The relative error between the two technologies is only $1.8 \%$. To further confirm the wide measurement range of the IJ-FPGA technology, we also measured $\sim 100$ ps-level ultrashort pulses at $1.06 \mu \mathrm{m}$. The experimental setup of such $\sim 100$ ps-level $1.06 \mu \mathrm{m}$ laser is similar to Fig. 4(a), except that a SESAM with different performance was used. The central wavelength of the ultrafast laser is $1063.1 \mathrm{~nm}$, as shown in Fig. 4(d), and the pulse repetition rate is $18.601 \mathrm{MHz}$.
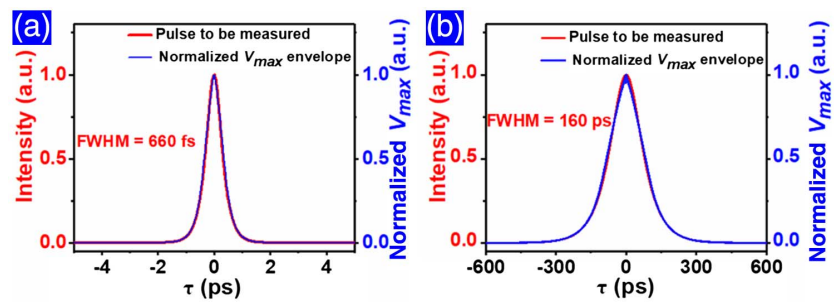

Fig. 3. Intensity envelope of the original pulse (red line) and the numerically simulated $\left(\tau, V_{\text {max }}\right)$ envelope (blue line) in (a) the fs regime and (b) the ps regime.
When the input average power is $62 \mu \mathrm{W}$, in Fig. 4(e), the pulse widths measured by our IJ-FPGA setup and a commercial autocorrelator are 81.53 ps and 81.65 ps, respectively. The relative error is as low as $0.15 \%$.

Furthermore, to verify the broadband spectrum applicability of the IJ-FPGA technology, we used the setup to measure $1.5 \mu \mathrm{m}$ and $2.15 \mu \mathrm{m}$ ultrashort pulses as follows. Figure 5(a) is a
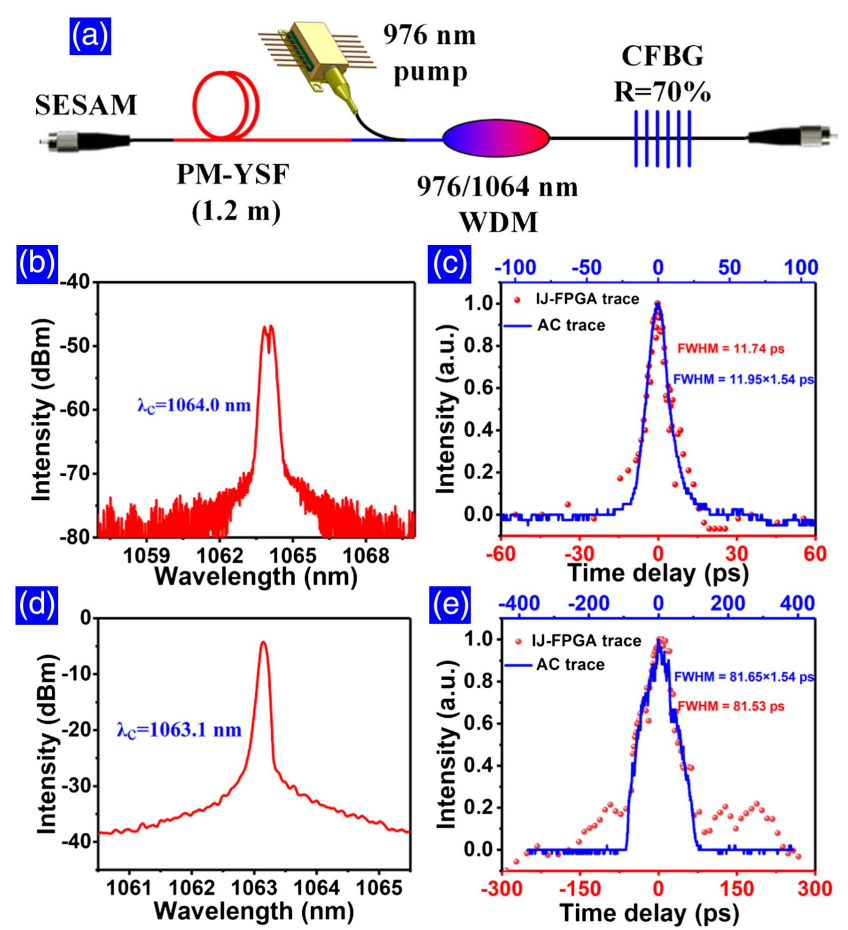

Fig. 4. (a) Experimental setup of the $1.06 \mu \mathrm{m}$ ultrafast laser. WDM, wavelength-division multiplexer; PM-YSF, polarization-maintaining Yb-doped fiber; CFBG, chirped fiber Bragg grating. (b) and (d) Optical spectra. (c) and (e) AC trace with a commercial autocorrelator (blue line) and IJ-FPGA trace (red dots). 

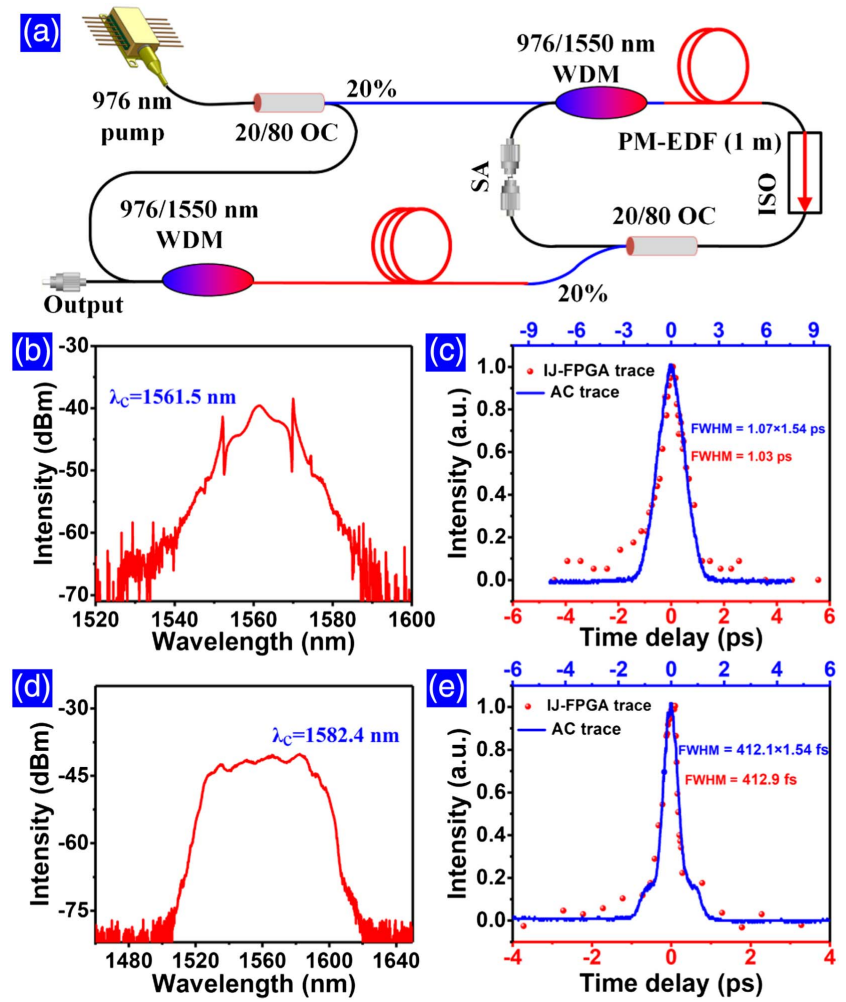

Fig. 5. (a) Experimental setup of the $1.5 \mu$ m ultrashort pulsed laser. OC, optical coupler. (b) and (d) Optical spectra of the measured ultrafast laser. (c) and (e) AC trace with a commercial autocorrelator (blue line) and IJ-FPGA trace (red dots).

homemade $1.5 \mu \mathrm{m}$ CNT mode-locked polarization-maintaining Er-doped fiber laser, and the laser has a central wavelength of $1561.5 \mathrm{~nm}$ [see Fig. 5(b)] and a pulse repetition rate of $22.801 \mathrm{MHz}$. Figure 5(c) gives the pulsewidth measured result (red dots) by the IJ-FPGA setup with the input average power of $5 \mu \mathrm{W}$, and $\Delta \tau^{\mathrm{FWHM}}=1.03$ ps. The blue line in Fig. $5(\mathrm{c})$ was measured by the FR-103XL commercial autocorrelator, and the pulse width is $1.07 \mathrm{ps}$. The measured results of both technologies are in good agreement, and the relative error is $3.8 \%$. Then, we also measured fs pulses at $1.58 \mu \mathrm{m}$ (based on nonlinear spectral broadening). The central wavelength is $1582.4 \mathrm{~nm}$ with a $3 \mathrm{~dB}$ bandwidth of $\sim 55 \mathrm{~nm}$ [see Fig. 5(d)] and a pulse repetition rate of $14.293 \mathrm{MHz}$. As shown in Fig. 5(d), when the input average power was $20 \mu \mathrm{W}$, the pulsewidth measured by our IJ-FPGA setup and the FR-103XL commercial autocorrelator is $412.9 \mathrm{fs}$ and $412.1 \mathrm{fs}$, respectively. The experimental results well prove the reliability of the IJ-FPGA for measuring fs ultrashort pulses. However, it should be pointed out that the measurement accuracy will sharply worsen for $<200$ fs pulsewidth, due to the nonlinearity and dispersion influence of fiber used in the IJ-FPGA setup on pulsewidth broadening or compression.

We further utilized the IJ-FPGA technology to measure an ultrafast laser operated at $2.15 \mu \mathrm{m}$. Figure 6(a) depicts a schematic of the $2.15 \mu \mathrm{m}$ ultrafast laser based on Raman soliton self-frequency shift. As shown in Fig. 6(b), the $2153.0 \mathrm{~nm}$
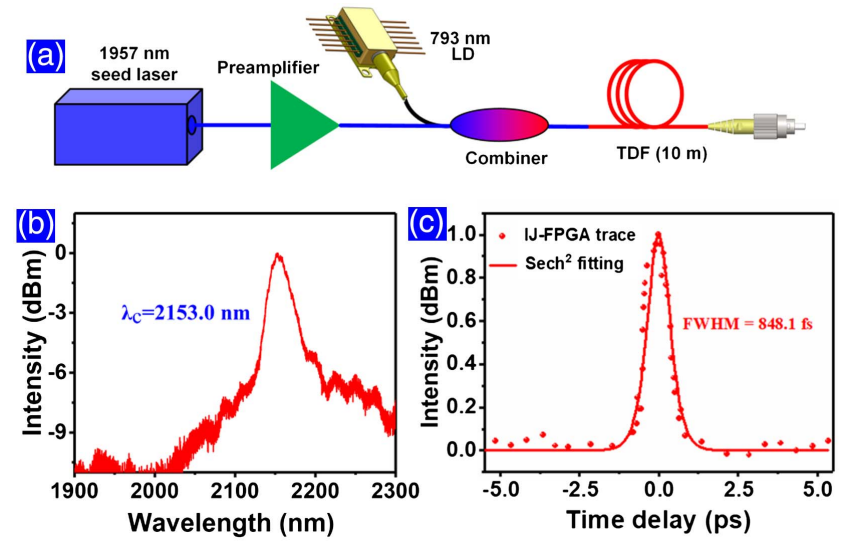

Fig. 6. (a) Schematic of the $2.15 \mu \mathrm{m}$ ultrafast fiber laser. TDF, $\mathrm{Tm}^{3+}$-doped double-clad fiber. (b) Optical spectrum. (c) IJ-FPGA trace (red dots) and fitting (red line).

ultrashort pulsed laser has a $3 \mathrm{~dB}$ bandwidth of $39.0 \mathrm{~nm}$, and the pulse repetition rate is $35.305 \mathrm{MHz}$. In the Fig. 6(c), the red dots were recorded by the IJ-FPGA setup at the input power of $150 \mu \mathrm{W}$. By sech ${ }^{2}$ fitting, the pulsewidth was measured as $\Delta \tau^{\mathrm{FWHM}}=848.1 \mathrm{fs}$, but the AC result for comparsion cannot be provided due to the inaccessibility of the FR-103XL autocorrelator at $2.15 \mu \mathrm{m}$.

\subsection{High-sensitivity pulsewidth measurement}

In addition, to evaluate the measurement sensitivity of the IJ-FPGA technology, a tunable optical attenuator was inserted to the output end of a homemade $1.56 \mu \mathrm{m}$ ultralfast laser to repeat the pulsewidth measurement with the different input average power. The pulse widths of the ultrafast laser with average input power of $10 \mu \mathrm{W}, 500 \mu \mathrm{W}$, and $2 \mathrm{~mW}$ were measured by the IJ-FPGA setup, respectively. The results are shown in Fig. 7, and all of them are the same as $613.7 \mathrm{fs}$, confirming that the IJ-FPGA technology can be valid in a wide range [from microwatts $(\mu \mathrm{W})$ to milliwatts $(\mathrm{mW})$ at least] of input power and shows an excellent consistency in pulsewidth measurement.

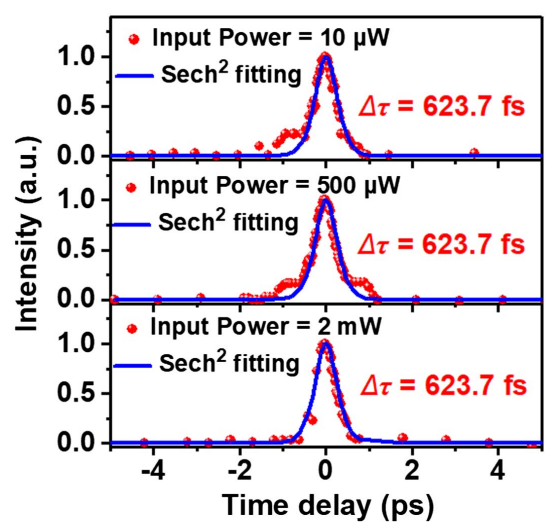

Fig. 7. IJ-FPGA trace (red dots) and fitting envelope (blue line) with different input power (pulse energy). 
Last, we have calculated the average-power-peak-power product $\left(P_{\mathrm{av}} \cdot P_{\mathrm{pk}}\right)$ and the measurable pulse energy in the 1.06$2.15 \mu \mathrm{m}$ wavebands. A minimum pulse energy of $219 \mathrm{fJ}$ is experimentally detected with $P_{\mathrm{av}} \cdot P_{\mathrm{pk}}$ of $1.065 \times 10^{-6} \mathrm{~W}^{2}$. Compared with the detected $P_{\mathrm{av}} \cdot P_{\mathrm{pk}}$ of the FR-103XL commercial autocorrelator (i.e., $\sim 10^{-3} \mathrm{~W}^{2}$ in our experiment), the minimum detectable $P_{\mathrm{av}} \cdot P_{\mathrm{pk}}$ of our IJ-FPGA setup is remarkably improved, i.e., a higher sensitivity. In fact, the sensitivity of the IJ-FPGA setup is limited by the large loss $(3.98 \mathrm{~dB}$ at $1.5 \mu \mathrm{m}$ and $6.99 \mathrm{~dB}$ at $1.06 \mu \mathrm{m}$ ) of the delay line used in our system. If the whole fiber system is fusion spliced instead of using fiber connectors, and a better delay line with low insertion loss is chosen, we estimate that the allowable single pulse energy can be $\sim 88 \mathrm{fJ}$, and the $P_{\mathrm{av}} \cdot P_{\mathrm{pk}}$ can be improved to $1.7 \times 10^{-7} \mathrm{~W}^{2}$ (corresponding to an average power of $-23.01 \mathrm{dBm}-3.98 \mathrm{~dB}=$ $-26.99 \mathrm{dBm})$ in the $1.5 \mu \mathrm{m}$ band. In the $1.06 \mu \mathrm{m}$ band, the allowable single pulse energy can be estimated as $\sim 155 \mathrm{fJ}$, and the $P_{\mathrm{av}} \cdot P_{\mathrm{pk}}$ can be improved to $3.96 \times 10^{-8} \mathrm{~W}^{2}$ (corresponding to an average power of $-18.24 \mathrm{dBm}-6.99 \mathrm{~dB}=-25.23 \mathrm{dBm}$ ).

\section{Conclusion}

In summary, we have proposed and demonstrated a ultrafast laser pulsewidth measurement technology based on IJ-FPGA. This technology does not need nonlinear crystals or a TPA detector and shows a compact structure and intelligent capability. By using the IJ-FPGA technology, we have measured the pulsewidths of the ultrafast lasers at 1.06, 1.5, and $2.15 \mu \mathrm{m}$ wavelengths, and the measurement results are consistent with a commercial autocorrelator and show a small relative error of $0.15 \%-3.8 \%$, verifying the feasibility of the IJ-FPGA technology. A minimum pulse energy of $219 \mathrm{fj}$ is experimentally detected with a $P_{\mathrm{av}} \cdot P_{\mathrm{pk}}$ of $1.065 \times 10^{-6} \mathrm{~W}^{2}$. By further optimizing the experimental setup, it is estimated that the system can potentially support femtojoule (fJ)-level pulse energy with fs pulse width, and the $P_{\mathrm{av}} \cdot P_{\mathrm{pk}}$ can be improved to $10^{-8} \mathrm{~W}^{2}$. We believe that the IJ-FPGA technology has the potential to achieve a miniaturized, high-sensitivity, and broadband pulsewidth measurement system.

\section{Acknowledgement}

This work was supported by the National Science Fund for Excellent Young Scholars (No. 62022069), the Fundamental Research Funds for the Central Universities (No. 20720200068), and the Shenzhen Science and Technology Project (No. JCYJ20210324115813037).

${ }^{\dagger}$ These authors contributed equally to this work.

\section{References}

1. M. Wojtkowski, A. Kowalczyk, R. Leitgeb, and A. F. Fercher, "Full range complex spectral optical coherence tomography technique in eye imaging," Opt. Lett. 27, 1415 (2002).
2. K. N. Joo and S. W. Kim, "Absolute distance measurement by dispersive interferometry using a femtosecond pulse laser," Opt. Express 14, 5954 (2006).

3. J. H. Bruning, "Digital wavefront measuring interferometer for testing optical surfaces and lenses," Appl. Opt. 13, 2693 (1974).

4. J. J. Zondy, D. Kolker, C. Bonnin, and D. Lupinski, "Second-harmonic generation with monolithic walk-off-compensating periodic structures. II. Experiments," J. Opt. Soc. Am. B 20, 1695 (2003).

5. N. Pontius, A. V. Neeb, W. Eberhardt, G. Lüttgens, and P. S. Bechthold, "Ultrafast relaxation dynamics of optically excited electrons in $\mathrm{Ni}_{3}^{-}$," Phys. Rev. B 67, 106 (2003).

6. D.-N. Wang, "Review of femtosecond laser fabricated optical fiber high temperature sensors [Invited]," Chin. Opt. Lett. 19, 091204 (2021).

7. M. L. M. Balistreri, H. Gersen, J. P. Korterik, L. Kuipers, and N. F. van Hulst, “Tracking femtosecond laser pulses in space and time," Science 294, 1080 (2001).

8. H. P. Weber, "Method for pulsewidth measurement of ultrashort light pulses generated by phase-locked laser using nonlinear optics," J. Appl. Phys. 38, 2231 (1967).

9. D. J. Kane and R. Trebino, "Characterization of arbitrary femtosecond pulses using frequency-resolved optical gating," IEEE J. Quantum Electron. 29, 571 (1993).

10. R. Trebino, K. W. Delong, D. N. Fittinghoff, J. N. Sweetser, M. KrumbüGel, B. A. Richman, and D. J. Kane, "Measuring ultrashort laser pulses in the time-frequency domain using frequency-resolved optical gating," Rev. Sci. Instrum. 68, 3277 (1997).

11. S. D. Yang, A. M. Weiner, K. R. Parameswaran, and M. M. Fejer, "400-photon-per-pulse ultrashort pulse autocorrelation measurement with aperiodically poled lithium niobate waveguides at $1.55 \mu \mathrm{m}$," Opt. Lett. 29, 2070 (2004).

12. D. N. Fittinghoff, J. L. Bowie, J. N. Sweetser, R. T. Jennings, and I. A. Walmsley, "Measurement of the intensity and phase of ultraweak, ultrashort laser pulses," Opt. Lett. 21, 884 (1996).

13. J. Gagnon, E. Goulielmakis, and V. S. Yakovlev, "The accurate FROG characterization of attosecond pulses from streaking measurements," Appl. Phys. B 92, 25 (2008).

14. C. Iaconis and I. A. Walmsley, "Spectral phase interferometry for direct electric-field reconstruction of ultrashort optical pulses," Opt. Lett. 23, 792 (1998).

15. G. Stibenz and G. Steinmeyer, "Optimizing spectral phase interferometry for direct electric-field reconstruction,” Rev. Sci. Instrum. 77, 073105 (2006).

16. J. Bromage, C. Dorrer, I. A. Begishev, N. G. Usechak, and J. D. Zuegel, "Highly sensitive, single-shot characterization for pulse widths from 0.4 to 85 ps using electro-optic shearing interferometry," Opt. Lett. 31, 3523 (2006).

17. A. Hayat, A. Nevet, P. Ginzburg, and M. Orenstein, "Applications of twophoton processes in semiconductor photonic devices: invited review," Semicond. Sci. Technol. 26, 083001 (2011).

18. U. Steinmeyer, "A review of ultrafast optics and optoelectronics," J. Opt. A 5, R1 (2003).

19. W. Tawfik, "Precise measurement of ultrafast laser pulses using spectral phase interferometry for direct electric-field reconstruction," J. Nonlinear Opt. Phys. Mater. 24, 1550040 (2015).

20. J. K. Ranka, A. L. Gaeta, A. Baltuska, M. S. Pshenichnikov, and D. A. Wiersma, "Autocorrelation measurement of 6-fs pulses based on the two-photon-induced photocurrent in a GaAsP photodiode," Opt. Lett. 22, 1344 (1997).

21. J. M. Roth, T. E. Murphy, and C. Xu, "Ultrasensitive and high-dynamic-range two-photon absorption in a GaAs photomultiplier tube," Opt. Lett. 27, 2076 (2002).

22. E. Z. Chong, T. F. Watson, and F. Festy, "Autocorrelation measurement of femtosecond laser pulses based on two-photon absorption in GaP photodiode," Appl. Phys. Lett. 105, 062111 (2014).

23. P. Xiao, K. Wu, D. Mao, and J. Chen, "A pulsewidth measurement technology based on carbon-nanotube saturable absorber," Opt. Express 27, 4188 (2019).

24. J. Chen, M. Wang, and W. Xia, "Neural-network-assisted femtosecond laser pulse duration measurement using two-photon absorption," Chin. Opt. Lett. 18, 121901 (2020). 
25. D. T. Reid, W. Sibbett, J. M. Dudley, L. P. Barry, B. Thomsen, and J. D. Harvey, "Commercial semiconductor devices for two photon absorption autocorrelation of ultrashort light pulses," Appl. Opt. 37, 8142 (1998).

26. G. Cong, O. Makoto, M. Yuriko, O. Morifumi, and Y. Koji, "Interferometric autocorrelation of ultrafast optical pulses in silicon sub-micrometer p-i-n waveguides," Opt. Express 26, 15090 (2018).

27. M. Valadan, D. D'Ambrosio, F. Gesuele, R. Velotta, and C. Altucci, "Linear optical methods for temporal characterization of femtosecond UV pulses," Proc. SPIE 9135, 91350G (2014).
28. X. Shen, J. Liu, F. Li, P. Wang, and R. Li, "Extended transient-grating self-referenced spectral interferometry for sub-100 nJ femtosecond pulse characterization," Chin. Opt. Lett. 13, 081901 (2015).

29. X. Shen, P. Wang, J. Liu, and R. Li, "Compact transient-grating selfreferenced spectral interferometry for sub-nanojoule femtosecond pulse characterization," Appl. Opt. 56, 582 (2017).

30. M. Miranda, C. L. Arnold, T. Fordell, F. Silva, B. Alonso, R. Weigand, A. L'Huillier, and H. Crespo, "Characterization of broadband few-cycle laser pulses with the d-scan technique," Opt. Express 20, 18732 (2012). 